\title{
PREVALENCE, CLINICAL FEATURES AND FACTORS \\ ASSOCIATED WITH TOOTH WEAR \\ IN THE UKRAINIAN YOUNG ADULT POPULATION
}

\section{Fastovets Olena ${ }^{1}$}

DOI: http://dx.doi.org/10.30525/978-9934-571-31-2_11

Abstract. Tooth wear in young individuals has increased significantly in recent years. Thus, the purpose of the present study is to estimate the prevalence and to learn clinical features and factors associated with tooth wear in the Ukrainian adult population age to 40 years. Methodology. Data on clinical investigation were collected in a survey of 2053 employees of ore mining enterprise aged 18-40 years. It has been studied the prevalence and clinics of tooth wear depending on age and sex, and also taking into account influencing of the working-environment harmful and other etiological factors. The investigation involved the analysis of tooth contacts on working and balancing sides and the definition of type of intercuspidation which is described by occlusal concepts. Results. According to the obtained data, the prevalence of tooth wear was $27.6 \pm 1.0 \%$. The process was the most severe in spreading and depth of loss in the presence of working-environment harmful factors $(32.1 \pm 1.5 \%$ vs. $23.4 \pm 1.3 \%, \mathrm{p}<0.05)$. The absence of any strong correlation between progressive tooth wear and such etiological factors as age $(\mathrm{r}=0.48 ; \mathrm{p}<0.05)$, work experience in harmful production $(\mathrm{r}=0.61$; $\mathrm{p}<0.05)$, the dust content in air of the working zone $(\mathrm{r}=0.63 ; \mathrm{p}<0.05)$, the presence of background pathology of the gastrointestinal tract $(\mathrm{r}=0.42$; $\mathrm{p}<0.05)$ and endocrine diseases $(\mathrm{r}=0,18, \mathrm{p}>0.05)$, the presence of local factors $(r=0.38 ; p<0.05)$, excessive oral hygiene $(r=0.12 ; p<0.05)$, use of carbonated drinks $(r=0.32 ; \mathrm{p}<0.05)$ suggested the impossibility of providing a leading trigger mechanism for the development of the tooth wear. As a result of the clinical study of dental contacts in lateral occlusion, it was found that $30.0 \pm 3.7 \%$ persons had canines' contacts and $70.0 \pm 3.7 \%$ had group contacts of canines, premolars and molars under physiological tooth wear. Otherwise, in the group of pathological (increased) tooth wear, canines' con-

\footnotetext{
${ }^{1}$ Doctor of Medical Sciences, Associate Professor, Head of Department of Prosthetic Dentistry,

State Establishment "Dnipropetrovsk Medical Academy of Ministry of Health of Ukraine", Ukraine 


\section{Fastovets Olena}

tact on the working side was established in $23.5 \pm 4.2 \%$ of cases, group contacts $-76.5 \pm 4.2 \%$. Thus, significant differences between the prevalence of occlusal concepts in the research group had not been found $(p>0.05)$. Intercuspidation on balancing side was found in $36.0 \pm 3.9 \%$ of persons who have physiological tooth wear, and $63.3 \pm 4.8 \%$ - pathological one $(\mathrm{p}<0.05)$. Significant difference between the prevalence of bilateral balancing occlusion in research groups demonstrated that these occlusal contacts promoted increased wear of occlusal surfaces, especially chewing segments of dentition. Thus, dispensary observation of patients with tooth wear should take into account the clinical and pathogenetic features of the disease, comply with the principles of complexity of medical and diagnostic measures, fulltime care, stage and preventive direction. Conclusion. Tooth wear is a prevalent condition in this population. Significantly higher prevalence and severity of tooth wear was associated with the exposure of harmful factors of dust-gas mixture from the production. In addition, other etiological factors contribute to the development of the pathological process in enamel and dentine, among which peculiarities of occlusal contacts, described as "occlusal concepts". To reduce the prevalence and intensity of tooth wear, in particular in the adult population age to 40 years, there is a need for dispensary, which involves early diagnosis, regular examinations, prophylaxis and treatment, aimed at preventing complications.

\section{Introduction}

Tooth wear has an increasing prevalence all over the word [1, p.548; 2 , p.88; 3, p. 50]. This problem is complex, because no evidence-based guidelines are available, the clinician may have difficulties deciding which treatment option to choose to resolve difficult situations [4, p. 757].

Nowadays, it's known that tooth wear can be the result of a natural ageing process and be imperceptible to the majority of the patients. However, in some young patients the pathogenesis of tooth wear is unclear. The disease has special clinical features as progressive character and tooth hypersensitivity. Severe forms of tooth wear may extend to the pulp. In the absence of adequate treatment the generalized tooth wear has been reported to decrease occlusal vertical dimension with temporomandibular disorder-associated symptoms [5, p. 2].

Clinically tooth wear is easy for diagnostics; it defined as loss of enamel and dentine by a chemical or mechanical process not involving bacteria. 
With this, tooth facets are characterized as flat, round or sharply angled and polished surfaces on the occlusal or incisal areas of the teeth. They preferentially affect the palatal surfaces of maxillary anterior teeth and occlusal surfaces of mandibular first molars [6, p. 20-21].

According to modern ideas, tooth wear is explains as a result of three processes: abrasion (wear produced by interaction between teeth and other materials), attrition (wear through tooth-tooth contact) and erosion (dissolution of hard tissue by acidic substances) [7, p. 33].

Erosion is associated with intrinsic or extrinsic acids, and therefore, subjects with reflux disease and eating disorders are at increased risk. Fruit juice, fruits and carbonated drink consumption, frequency of consumption and specific habits are also risk factors. Attrition is more prevalent under bruxism. Other habits need to be considered when defining the risk of tooth wear. The way of the mandibular teeth contact with the maxillary teeth may influence tooth wear. Abrasion is usually associated with tooth brushing and toothpastes, especially in an already acidic environment [8, p. 26].

Individuals have different risks for developing tooth wear depending on their environment and occupation. It is anticipated that people with regular working-environment harmful factors such as: microclimate, dust, noise, vibrations, and chemical compounds have an especially high risk of developing tooth wear and more severe pathological process [13, p.10].

Because of its multifactorial aetiology and pathogenesis, tooth wear can manifest itself in many different representations, thus, it can be difficult to diagnose and manage the condition [9, p. 78].

The dental care evaluation highlights that improvements are required in recording, risk assessing and preventive treatment planning of tooth wear. Experienced dentists were less likely to risk assess tooth wear and less likely to provide preventive treatment $[10$, p. 83].

Also discussed will be the administration of preventative and passive management strategies for cases displaying tooth wear [11, p. 25].

At present, investigating the prevalence of tooth wear in young people has become an interesting and feasible proposal, since this pathology requires special attention. Therefore, it is necessary to conduct studies for following up and preventing this wear, in order to collect data and monitor the condition when it is present, and thus serve as a guide to future approaches [12, p. 1011]. 


\section{Fastovets Olena}

Moreover, there is no data on the prevalence of tooth wear in Ukraine, peculiarities of the morbidity in which are due to deterioration of the ecological situation. Besides, harmful production, where there are abrasive factors, may cause the risk of tooth wear development.

In our opinion, the study of tooth wear during a screening survey of the organized contingent of population, in our case they are the employees of ore mining enterprise, in an amount that would ensure the reliability of the results, would determine the prevalence and peculiarities of the disease in our region. This way, it helps to justify the directions of further research on improvement of diagnostic, therapy and prophylaxis of tooth wear.

Taking into consideration the foregoing discourse, the aim of the present study is to estimate the prevalence, to learn clinical features and factors associated with tooth wear in the Ukrainian adult population age to 40 years.

\section{Sample for clinical study}

The present study was conducted with 2053 employees of ore mining enterprise aged 18-40 years. The participants signed the free and informed term of consent and subsequently participated in the present investigation.

The exclusion criteria were as follows: individuals aged less 18 years and more 40 years; those who used orthodontic appliances because they made it difficult to perform clinical evaluation of the tooth surface. Criteria for inclusion in the study were as follows: individuals who were working at ore mining enterprise and patients who agree to participate in the research.

The sample size calculation was based on a study [14, p. 228]. From this datum, a margin of error of $5 \%$, level of confidence of $95 \%$, and power of $80 \%$ were established. Considering the adjustment for finite population, a sample " $n$ " was obtained. To allow for possible losses, $20 \%$ was added, leading to a final number of participants.

The participants were broken by two groups according the presence of working-environment harmful factors. In the first research group there were 997 individuals, who had harmful factors in the production, mainly dust and gaseous chemical compounds, as well as vibration and noise.

The remaining 1056 persons, who did not have any working-environment harmful factors, were included in the second research group.

The analysis was carried out in 3 age groups: up to 20 years, 20-29 years, $30-39$ years. In the age group up to 20 years there were 268 male $(68.7 \pm 2.3 \%)$ and 122 female $(31.3 \pm 2.3 \%)$; in the group $20-29$ years -512 male $(67.6 \pm 1.7 \%)$ 
and 122 female $(32.4 \pm 1.7 \%)$; in the group $30-39$ years -563 male $(62.1 \pm 1.6 \%)$ and 343 female $(37.9 \pm 1.6 \%)$.

Thus, the total number of men, included in the survey, was 1343 $(65.4 \pm 1.0 \%)$, women $-710(34.6 \pm 1.0 \%)$. In the first research group there were 793 male $(79.5 \pm 1.3 \%)$ and 204 female $(20.5 \pm 1.3 \%)$; in the second one -550 male $(52.1 \pm 1.5 \%)$ and 506 female $(47.9 \pm 1.5 \%)$. The majority of men in the first group is due to the specifics of the occupations of industrial workers. At the same time, the increase in the number of women in the second group is caused by the peculiarities of the professional composition of productions without harmful factors, usually management, servicing and auxiliary.

\section{Clinical examination}

Data on tooth wear was collected during the clinical examination. First of all, tooth wear within the enamel was considered physiological [6, p. 5].

For the description of the pathological (increased) tooth wear in hard tissues, it was used Bushan A. G. classification. The degrees of severity of tooth wear were classified in depth of loss as: up to one third of the height of the crown (the first degree); from one third to two thirds of the height of the crown (the second degree); from two thirds of the height of the crown (the third degree).

On the plane of the loss it was differentiated the horizontal, vertical and mixed forms of tooth wear. In the longevity of the pathological process, it was diagnosed the generalized and localized forms. About localized tooth wear it was said in case of damage to $25 \%$ of dentitions [6, p. 21-22].

According to Trezubov V. N., Shcherbakov A. S., Mishnev L. M., depending on the severity of the compensatory and adaptive reaction of the chewing apparatus, it were three clinical forms of tooth wear such as: uncompensated (with decrease occlusal vertical dimension), compensated (without decrease occlusal vertical dimension) and subcompensated (intermediate variant) [6, p. 23-24].

Finally, diagnosis of such a complication of tooth wear, like hyperesthesia of hard tissues, was carried out by determining the reaction to temperature stimuli.

Information on caries, periodontal disease and number of missing teeth was collected during chart review. Periodontal disease was considered present if the chart had any record of bone loss in the past 12 month. 


\section{Fastovets Olena}

The sample of background general diseases was performed according to the "Appendix 2 to the Regulations on the Medical Examination of Certain Categories of Employees" and the "List of Preventive Examinations". Particular attention was paid to those general diseases, which, according to the literature, are associated with the development of tooth wear [13, p. 9].

\section{Occlusal analysis}

In order to analyze the influence of occlusal concepts (features of closing of the teeth in lateral occlusion) on the character of tooth wear, we conducted an examination of 102 patients with pathological (increased) tooth wear and 150 individuals with a physiological form of tooth wear (as a comparison) under the age of 40 years. They were selected on the stage of clinical examination.

Participants were divided into three equal gender and age groups: 18-20, 20-29, 30-39 years, where women accounted for $56.3 \pm 3.1 \%$, men $43.7 \pm 3.1 \%$. To verify the nature of the tooth wear (physiological or pathological), the age of the patient and the intensity of the loss were compared, using Moldovanov's A.G. classification [6, p. 5]. All participants had intact dentitions and orthognathic bite.

The study of occlusal contacts was performed on the diagnostic models casted into articulator. The occlusal surfaces of tooth were investigated. The occlusal contacts on the working and balancing sides in lateral occlusion were described by occlusion concepts $[15$, p. 28].

For study the localization of occlusal contacts, the occlusal method according to Milikevich V. Yu. was used. Warmed denture wax, $1.2 \mathrm{~mm}$ thick, was placed on the lower dentition into the oral cavity. The patient asked to close his mouth in the position of central occlusion. After cooling, wax was removed from the oral cavity and put on the plaster models. The places of perforations on the occlusiogram were transferred using a marker to plaster models [16, p. 57].

\section{Statistical analysis}

The obtained results involved descriptive and inferential statistics. Descriptive statistics was used to describe the sample and the prevalence of tooth wear. The chi-square test was applied to evaluate association among the independent variables and the outcome (tooth wear). To investigate the explanatory factors for tooth wear, the regression with robust variance was 
performed. The independent variables that presented $\mathrm{p}<0.05$ in the univariate model were inserted into the multivariate regression model. The level of significance adopted was $5 \%$.

For the quantitative characteristics of the correlation, physiological tooth wear was conventionally considered to be 0 ; increased tooth wear within the enamel and partly dentin - as 1; within the base dentin - as 2; within the limits of substitute dentin - as 3. The presence of background general diseases and local factors that can cause wear of enamel and dentin, indicated as 1 , absence - as 0 .

In turn, the correlation coefficients ranged from -1 to +1 . At $r<0.3$, the correlation was considered weak, at $r=0.3-0.7$ - moderate, at $r>0.7-$ strong. At positive values of the correlation coefficient the relationship between the studied indicators was determined as direct, with negative - as inverse. For $r$ values of 0 , there was no linear relationship.

The software program MS Excel 2003 was used for the analyses.

\section{Prevalence and clinical features of tooth wear}

It was found that the prevalence of tooth wear among the employees of ore mining enterprise aged $18-40$ years was $27.6 \pm 1.0 \%$. It was established the significant difference in the prevalence of pathological process in the individuals from the first and second research groups. Thus, in employees, who have the harmful factors in working environment, the tooth wear was registered in $32.1 \pm 1.5 \%$ of cases vs. $23.4 \pm 1.3 \%$ for the second group.

There was increasing the prevalence of tooth wear according the age. So, the highest values were set for $30-39$ year olds $(41.0 \pm 1.5 \%$ for the first group and $29.2 \pm 1.4 \%$ for the second one). The prevalence of the pathological process at the age of 20-29 years was detected as $33.2 \pm 1.5 \%$ for the first group and $24.3 \pm 1.3 \%$ for the second one. At last, at the age under 20 the prevalence of tooth wear was minimal $(11.2 \pm 1.0 \%$ and $6.7 \pm 0.7 \%$ for the both groups respectively, $\mathrm{p}<0.05$ ).

In the first group, the prevalence of tooth wear was higher in men than in women, regardless of age, which was probably due to a higher level of harm factors in the work place $(29.6 \pm 1.6 \%$ vs. $24.0 \pm 3.0 \%, \mathrm{p}<0.05)$. In the second group, there were no significant gender differences for people under the age of $20(7.4 \pm 2.0 \%$ vs. $5.9 \pm 2.0 \%, p>0.05)$ and $20-29$ $(25.6 \pm 2.9 \%$ vs. $22.4 \pm 3.4 \%, \mathrm{p}>0.05)$, while between $30-39$ year-old patients with the tooth wear were dominated by men $(34.9 \pm 3.3 \%$ vs. 


\section{Fastovets Olena}

$24.8 \pm 2.6 \%, p<0.05)$, although the greater severity of damage of enamel and dentin was observed in women.

Among patients with tooth wear, it was determined some predominance of local character of the pathological process. The local tooth wear was diagnosed in $57.8 \pm 2.1 \%$ of patients. Local tooth wear most often occurred in a horizontal plane, while the occlusion surfaces of molars and premolars were struck. The prevalence of this form was $57.3 \pm 2.1 \%$. Vertical form of tooth wear occurred in the area of the maxilla and mandible under deep bite $(0.5 \pm 0.3 \%)$. For generalized tooth wear the mixed form was more characteristic, which was observed under orthognathic bite in $35.4 \pm 2.0 \%$ of cases and under deep incisal overlap - in $1.4 \pm 0.5 \%$. In $5.3 \pm 0.9 \%$ of the persons, the generalized horizontal form of the disease was registered under direct bite.

The first degree of tooth wear was the most often; its prevalence was $80.6 \pm 1.7 \%$. The second degree was detected in $17.1 \pm 1.6 \%$ of cases of tooth wear, the third one - in $2.3 \pm 0.6 \%$. The most severe tooth wear in the spread and depth of the lesion was observed in the patients from the first group. But subjective symptomatology was recognized as more pronounced in the second research group, where the number of cases of hyperesthesia was significantly higher $(40.9 \pm 3.1 \%$ vs. $30.3 \pm 2.6 \%, \mathrm{p}<0.05)$, which was probably related to other mechanisms of the disease. Most likely, this contingent of patients was dominated by the erosive component of tooth wear, mainly related to changes in contemporary lifestyle (dietary habits, ingestion of beverages, drugs, oral hygiene measures, eating disorders), biological (general health, biofilm, saliva), chemical factors (buffer capacity of saliva, $\mathrm{pH}$, type of acid, presence of calcium, fluoride and phosphorous), capable of interfering in the frequency and severity of erosive wear characterized hyperesthesia of hard tissues [2, p. 85].

According to anamnestic data, the lion's share accounted for the generalized form of hypersensitivity of hard tissues $(71.2 \pm 3.2 \%)$. In the other cases, patients had hyperesthesia in the area of frontal teeth. The complaints of temporary pain response from temperature stimuli were provided in $90.9 \pm 2.0 \%$ of patients with hypersensitivity of enamel and dentin.

Decrease occlusal vertical dimension with temporomandibular disorder-associated symptoms was established in $66.4 \pm 4.5 \%$ of patients with tooth wear of the second and third degrees. The average increase in the interocclusal gap was $2.7 \pm 0.6 \mathrm{~mm}$, the facial features were poorly expressed. 
Concomitant chronic angulous heilitis was diagnosed only in one case, in patient with deep traumatic occlusion $(0.2 \pm 0.1 \%)$.

It was established, that $15.4 \pm 3.4 \%$ of patients with tooth wear had compensated form of the disease with the development of dental-alveolar elongation. In $18.2 \pm 3.7 \%$ of cases the subcompensated form of tooth wear was diagnosed. In the remaining patients tooth wear was combined with a slight decrease in the height of the lower third of the face.

Taking into account the role of local factors, that cause decrease in occlusal vertical dimension, we found that in $9.7 \pm 1.2 \%$ of patients with tooth wear there were defects of dentition. Among them, deformations of dentitions were defined in $3.7 \pm 0.8 \%$ of the total number of patients with tooth wear. They were mainly mesial and distal movements of teeth. The part of persons with intact dentitions was $70.9 \pm 1.9 \%$. In $33.9 \pm 2.0 \%$ of cases, defects of the crown parts of teeth, mainly molars, were detected. According to the preliminary examination, in $2.1 \pm 0.6 \%$ of patients with tooth wear, the development of the process could be caused by irrational prosthetics.

On the contrary, tooth wear in the vast majority of cases was diagnosed in patients with healthy periodontal tissues. However, in $7.6 \pm 1.1 \%$ of patients with tooth wear, the state of decompensation from periodontal tissues was diagnosed, which was characterized by the development of destructive changes and the appearance of pathological mobility of teeth. Caries combined with tooth wear in $34.9 \pm 2.0 \%$ of cases. Non-carious cervical lesions (abfraction) were found in $14.4 \pm 1.5 \%$ of patients with tooth wear, enamel hypoplasia - in $1.1 \pm 0.4 \%$. Then how, the other non-carious lesions were not diagnosed.

It should be noted, that only $6.5 \pm 1.0 \%$ of patients with tooth wear applied for a dentist about the disease, although $12.9 \pm 1.4 \%$ of them needed already prosthetic treatment which includes increase occlusal vertical dimension in the complex of tertiary prophylaxis, aimed at the prevention of temporomandibular and chewing muscles disorders. $34.9 \pm 2.0 \%$ of patients needed conservative treatment of hyperesthesia. Accordingly, in the rest of the patients there was a need for prevention of further progressive tooth wear.

\section{Occlusal concepts}

Taking into account the fact that the occlusal disorders are the recognized prerequisite for the tooth wear $[15$, p. 8], it was analyzed the effect of 


\section{Fastovets Olena}

occlusive concepts (closing the teeth in lateral occlusion) to the tooth wear character.

In this work it was analyzed 273 occlusiograms. In particular, it was carried out a detailed analysis of the number and location of dental contacts and facets of wear on the teeth of the mandible and maxilla in order to determine the nature of tooth wear - physiological or increased (pathological).

According the results of the study of occlusal surfaces of teeth of patients of the age group up to 20 years, facets of wear were not detected. Contact areas were formed only. On chewing surfaces of the upper premolars there were diagnosed 2-3 such contact areas, which on occlusiograms had the appearance of areas of maximum thinning of the wax plate. In $41.3 \pm 6.2 \%$ of cases, there were 2 occlusal contacts, located on the medial and distal inner surfaces of the buccal cusps. Occlusion areas at the tops of the palatal cusps of the upper premolars were determined in $25.4 \pm 5.5 \%$ of cases. On the lower premolars, 2 occlusal contacts were located on the outer surface of the buccal cusps. The third contact area was found on medial slopes of lingual cusps only in $76.9 \pm 11.7 \%$ of patients with tooth wear. The upper molars had one less contact than the lower molars.

At the same time, if at the age of 20 years the contact areas on the chewing teeth were located on the medial surfaces of the occlusive slopes of the cusps, then at the age of 20-29 years, they were mainly in the middle of occlusal surfaces. In this age group, under the physiological tooth wear, there were both contact areas and facets of wear. On the chewing teeth of the upper and lower jaws, as well as on the lower canines and incisors, their localization was the same, but on the canines and incisors of the maxilla was different. The facets of wear on the upper canines, which appeared again, were located on the tearing cusp.

Under increased tooth wear in the age group of 20-29 years, on the lower incisors appeared contact areas, localized along the cutting edge. On the upper ones they were located on the medial and distal palatine rollers. There was a tendency to increase the area, described for the physiological tooth wear, and the creation of new ones on the distal-occlusal slopes of the palatine cusps of premolars and molars of the maxilla, as well as on the distal-occlusal slopes of the buccal cusps of the lower molars.

At the age of 30-39 years, physiological tooth wear were characterized by a shift of facets of wear from the tops of the buccal cusps to their outer slopes on the lower chewing teeth and from the tops of the palatine 
cusps to the inner slopes on the upper molars. New wear areas appeared on the so-called retrusive and protrusive surfaces of the upper and lower chewing teeth.

In the patients of the third age group there were significant differences for the pathological form of tooth wear in comparison with physiological one, the main of which was the presence of almost complete plane of contact between occlusal surfaces. On the upper incisors facets were located in a horizontal plane along the entire palatine surface, on the lower ones they were in a horizontal plane with a vestibular inclination. The horizontal facet of wear on tearing humps of upper canines merged with the vertical, which formed on a distal palatine surface. On the lower canines, tearing cusp flashed in a horizontal plane with a vestibule-distal inclination.

It should be noted that the generalized horizontal form of pathological tooth wear under orthognathic bite was characterized by a shallow incisor overlap, to $1 / 3$ of the length of the crowns of the lower anterior teeth, that was, on average 1-2 $\mathrm{mm}$. In this case, on the palatine surface of the upper incisors, and sometimes and the canines, facets of wear were formed, directed at different angles to the horizontal plane. With a deeper incisor overlap, a mixed form of tooth wear was diagnosed, which was manifested by more intensive wear of the chewing teeth in the horizontal plane, and less than the depth of loss of the anterior teeth in the vertical plane.

The occlusal surfaces of premolars and molars were characterized by the merger of separate areas of wear between themselves and the disappearance of second-order fissures with the preservation of the central fissures in the area of their transition to the buccal and lingual surfaces. The obtained data of character of tooth wear on the chewing surface allowed to determine the most widespread localization of the faces of wear, namely the lingual surface of the lower molars.

According to our observations, in the tooth wear of the first degree under orthognathic bite, the first molars and incisors on both jaws were the most often worn $(68.4 \pm 2.0 \%$ and $61.0 \pm 2.1 \%$ of cases respectively). The next ones were the premolars $(56.5 \pm 2.2 \%)$, finally they were the second molars $(51.3 \pm 2.2 \%)$ and canines $(44.7 \pm 2.2 \%)$. The third molars $(19.0 \pm 1.7 \%)$ are the least frequently worn. In direct bite, first of all, the frontal group of teeth was damaged $(60.0 \pm 7.7 \%)$. Under deep incision and deep bite tooth wear were formed in the first turn on the vestibular surfaces of the lower anterior teeth $(72.7 \pm 13.4 \%)$. 


\section{Fastovets Olena}

In turn, according to the results of the study of occlusal contacts in the lateral occlusion, it was found that in $30.0 \pm 3.7 \%$ of patients with physiological tooth wear it were canine guidance, and in $70,0 \pm 3,7 \%$ it was diagnosed group contacts of canines, premolars and molars. In the group of pathological tooth wear, the part of contact of canines on the working side, respectively, was $23.5 \pm 4.2 \%$, group contacts $-76.5 \pm 4.2 \%$. As shown, the reliable differences between the prevalence of any occlusal concept in experimental groups are not established ( $\mathrm{p}>0.05)$.

Intercusping on the balancing side was detected in $36.0 \pm 3.9 \%$ of individuals who had physiological tooth wear and in $63.3 \pm 4.8 \%$ of cases pathological $(\mathrm{p}<0.05)$. The reliable difference between the prevalence of bilateral balancing occlusion in patients with physiological and pathological tooth wear indicated that this occlusive contact contributed to the development of increased wear of chewing surfaces, first of all, lateral segments of dentitions.

Hyperbalancing contacts had not been established both under physiological and pathological tooth wear, which was probably due to intact dentitions and orthognathic bite.

Consequently, according to the obtained data, the features of closing the teeth in lateral occlusion can contribute to pathological (increased) tooth wear at young age. Proceeding from this, the diagnostic examination of interdental contacts, which involves the determination of indications for optimization of occlusion, will prevent the development or progression of the pathological process. Accordingly, the obligatory condition for the reproduction of optimal functional occlusion in the pathological tooth wear should be the study of its previous simulation in the articulators, necessary to achieve occlusal harmony, which involves the creation of not only stable multiple center contacts of the lateral teeth, but also protrusion and lateral guiding functions.

\section{Factors associated with tooth wear}

Using correlation-regression analysis, we evaluated the influence of different etiological factors in the pathogenesis of tooth wear.

As for the age aspect, as mentioned earlier, the highest frequency of the disease was found in persons aged 30-39. However, a significant jump in the prevalence of pathological process was found at the age of 20-29 years, while then there was not so much increase in the number of cases, but the deteriora- 
tion of the course of the tooth wear. Correlation between age and tooth wear was described as direct and moderate in strength $(\mathrm{r}=0.48, \mathrm{p}<0.05)$.

The predominance of tooth wear in employees with harmful industry factors indicated an unquestionable abrasive dust action. The mathematical estimation of this dependence proved the direct, close to strong, correlation between tooth wear and dust content in the air of the working zone $(\mathrm{r}=0.63$, $\mathrm{p}<0.05$ ). With the help of regression analysis, we calculated the threshold concentrations in relation to the threat of tooth wear. We calculated, that $4.7 \mathrm{mg} / \mathrm{m}^{3}$ is a critical concentration, under which, even in the absence of other etiological factors, tooth wear occurs. In turn, the dust content in the air of the working area is more than $1.1 \mathrm{mg} / \mathrm{m}^{3}$ increases the probability of tooth wear.

To increase the probability of tooth wear could be with work experience in harmful production. In the course of statistical processing between the tooth wear and the experience of harmful production, the direct, moderate correlation (close to strong) was established $(r=0.61 ; p<0.05)$.

Otherwise, in the second group, where the direct impact of damaging dust was almost eliminated, the prevalence of tooth wear was quite high, which indicated other causes of the pathological process. For their establishment, we conducted the analysis of data on general diseases in patients with tooth wear in the both groups. It was found that $39.5 \pm 2.7 \%$ of patients from the first research group and $47.8 \pm 3.2 \%$ of the second one had one or another background disease, indicating the possible overall progress of the loss of enamel and dentin.

According to our observations, the large part of background disease was associated with gastrointestinal tract disorders. Among them chronic gastritis, gastric ulcer and duodenal ulcer were prevalent. Their frequency among patients with tooth wear was $15.0 \pm 1.5 \%$ and $13.8 \pm 1.4 \%$ respectively. In the course of statistical processing, we have established a direct, moderate correlation between the degree of tooth wear and the presence of diseases of gastrointestinal tract $(\mathrm{r}=0.42, \mathrm{p}<0.05)$.

In assessing the influence of local pathogenetic factors, it was found that among patients with tooth wear, orthognathic bite was in $92.8 \pm 1.1 \%$, direct - in $5.3 \pm 0.9 \%$, deep - in $0.5 \pm 0.3 \%$, deep cutter overlap - in $1.4 \pm 0.5 \%$. In $9.7 \pm 1.2 \%$ of patients, defects of dental arteries were detected. The percentage of persons with intact dentitions was $70.9 \pm 1.9 \%$. In $33.9 \pm 2.0 \%$ of cases there wear defects of the crown parts of tooth. 


\section{Fastovets Olena}

According to the preliminary examination, in $2.1 \pm 0.6 \%$ of patients with tooth wear, the development of the pathological process could be caused by irrational dental prosthetics.

Localized form of increased tooth wear may be due to the presence of fillings on the chewing teeth. Wearing of filling material caused increase the chewing load on the intact teeth. In this case, there was loss of occlusal surfaces, interdental contacts, especially the support cusps. The connection of local factors and processes of tooth wear was moderate $(\mathrm{r}=0.38, \mathrm{p}<0.05)$.

Lastly, we conducted the analysis on the use of carbonated drinks, whose role in the development of erosive tooth wear, according literature, is considerable important. Emphasizing the subjectivity of received information, this factor associated with tooth wear took place only in $19.8 \pm 1.7 \%$ of patients $(r=0.32, p<0.05)$. Excessive oral hygiene, which wouldcauseso-called "oral-hygiene-relatedabrasionlesions", wasrecordedin $12.9 \pm 1.4 \%$ of cases $(\mathrm{r}=0.12, \mathrm{p}<0.05)$.

The presence in the anamnesis and in the results of a clinical study of several etiological factors was found in $31.9 \pm 2.0 \%$ of cases of tooth wear.

To sum up, according to our observations, dust and gaseous chemical compounds, as harmful production factors, can provoke the appearance and worsen the course of tooth wear. Their presence increased the probability of tooth wear in $8.7 \pm 0.6 \%$. Along with that, in their absence, the frequency of progressive form of tooth wear was also significant.

So, according to the results of the study, it has not been established the strong correlation between the tooth wear and any one associative factor, which indicates that it is impossible to isolate the leading trigger mechanism of the disease. Direct, moderate for strength of correlation, connections with a number of factors, indicate the polyetiological character of tooth wear.

In our opinion, it is expedient to differentiate the investigated etiological factors, not as exo- and endogenous, but by linking them with pathogenetic mechanisms, respectively, as those causing attrition, erosion and abrasion.

\section{Dispensary of patients with tooth wear}

The reduction of the frequency and severity of tooth wear, in particular among young people, can be achieved by means of dispensary - systematic medical surveillance for the purpose of early diagnostics, as well as timely treatment and prevention. In this regard, based on our research, we have 
developed organizational forms and methods of dispensary for patients with tooth wear.

First of all, the peculiarities of medical care of this contingent should be such as, firstly, the progressive nature of the loss of enamel and dentin, which leads to the inability to cure as a result of one-time and even multiple visits to the dentist; and secondly, the complexity of the early diagnosis of disease in the absence of quantitative criteria; thirdly, the severity of complications and the need for tertiary prophylaxis; fourthly, the high probability of background general diseases or factors that affect the clinic of tooth wear.

The formation of groups of "dynamic observation" occurs during preventive stomatological examinations.

Group D I includes healthy persons with physiological tooth wear and a favorable anamnesis. At the age of 25-40 years, wear of teeth of incisors and smooth out cusps of premolars and molars are normal.

Individuals under 40 years of age, who have signs of significant tooth wear, are recommended to hold up advanced clinical and laboratory research and dynamic follow up for three years in the group D II (practically healthy). This dispensary group is an intermediate link between healthy and sick. It includes persons with background diseases, which can affect tooth wear (diseases of the digestive tract, endocrine pathology, etc.). Individuals with working-environment harmful factors, such as abrasive (dust) and erosive (acids), should also be included into group D II.

Undoubtedly a significant role in the pathogenesis of tooth wear, bruxism and bruxomania plays. In the absence of clinical symptoms of tooth wear, patients with this pathology belong to group D II.

The anatomical and physiological preconditions that, under certain circumstances, can determine the pathogenetic links of progressive loss of enamel and dentin, in particular certain types of bite, also determine compliance with the second dispensary group.

The same should be attributed to patients with diagnosed tooth wear, but in the stage of long remission, that is, the stabilization of the process for three years and more. The purpose of observation of the dispensary group D II is the supportive treatment, aimed at preventing the progression of tooth wear.

Patients of the group D III are diagnosed with compensated form of tooth wear - loss of enamel and dentine that does not meet the age. Clinical 


\section{Fastovets Olena}

signs of decrease of occlusal vertical dimension are not expressed (distance between the frontal teeth does not exceed 6-8 $\mathrm{mm}$ ). In such cases, it is indicated appropriate conservative treatment, restoration of lost hard tissues and remineralization. But the main thing in this group is the systematic observation of occlusal vertical dimension. So, the purpose of dispensary is preventing disease transition to more severe form, and the task is to conduct adequate treatment and rehabilitation.

Group D IV is patients who require special medical supervision and prosthetic treatment and have a subcompensated and decompensated course of tooth wear. The purpose of the medical examination in them is the elimination of complications and the restoration of the lost tissues of the tooth. The task is the maintenance of treatment and rehabilitation measures. For this group, in checking examinations, the assessment of the state of dentures is of four integral criteria: aesthetics, functionality, restoration of the anatomical form of teeth, and the quality of the prosthetics. The favorable results of treatment of these patients should be considered not only a complete recovery, which is recorded in an average of $65 \%$ of cases, but also improving the condition, characterized by the restoration of the height of the lower face, chewing function and aesthetics.

Thus, each patient, at taking him to the dispensary register, needs a general stomatological examination and necessary for clarification of the diagnosis of laboratory and instrumental research. Then the plan of medical and recreational activities, which determines the frequency of observations and visits, terms of consultation, is drawn up.

Medical examinations, primary and regular (in-depth and control), allow to determine the diagnosis, recommend therapeutic and preventive measures, determine the effectiveness of past recommendations. In the framework of the dispensary, the diet is recommended for the patient, optimization of working conditions for harmful production, etc.

In general, in the diagnosis of patients with tooth wear, it is necessary to observe the general types of prophylaxis, formulated by WHO. Particular importance in preventing progressive tooth wear is given to oral hygiene. In turn, diet therapy should be considered not only as primary prophylaxis, but also as a measure of pathogenetic therapy.

In our opinion, the etiological and pathogenetic orientation of dispensary is a key to the success of prevention, detection of predisposition to the tooth wear, early diagnosis, which is a prerequisite for successful treatment. 


\section{Conclusions}

In summary, the conducted study has proven significant prevalence, as well as the tendency for "rejuvenation" of tooth wear among the young population of Ukraine. The prevalence of tooth wear was $27.6 \pm 1.0 \%$. It has been confirmed that dust and gaseous chemical compounds, as harmful production factors at ore mining enterprise, can provoke the appearance and worsen the course of tooth wear. The process was the most severe in spreading and depth of loss in the presence of working-environment harmful factors $(32.1 \pm 1.5 \%$ vs. $23.4 \pm 1.3 \%$ in their absence, $\mathrm{p}<0.05)$. Thus, their presence increases the prevalence of tooth wear on $8.7 \pm 0.6 \%$. Along with that, in the absence of working-environment harmful factors, the prevalence of tooth wear is also significant. More pronounced hyperesthesia pointed on probably erosive mechanisms of the disease.

The absence of any strong correlation between progressive tooth wear and such etiological factors as age $(\mathrm{r}=0.48 ; \mathrm{p}<0.05)$, work experience in harmful production $(\mathrm{r}=0.61 ; \mathrm{p}<0.05)$, the dust content in air of the working zone $(\mathrm{r}=0.63 ; \mathrm{p}<0.05)$, the presence of background disease of the gastrointestinal $\operatorname{tract}(\mathrm{r}=0.42 ; \mathrm{p}<0.05)$ and endocrine diseases $(\mathrm{r}=0,18, \mathrm{p}>0.05)$, the presence of local factors $(r=0.38 ; p<0.05)$, excessive oral hygiene $(r=0.12 ; p<0.05)$, use of carbonated drinks $(r=0.32 ; \mathrm{p}<0.05)$ suggests the impossibility of providing a leading trigger mechanism for the development of the disease.

It was found, $30.0 \pm 3.7 \%$ of individuals with physiological tooth wear had canines' contacts and $70.0 \pm 3.7 \%$ had group contacts of canines, premolars and molars. Otherwise, in the group of pathological (increased) tooth wear, canines' contact on the working side was established in $23.5 \pm 4.2 \%$ of cases, group contacts $-76.5 \pm 4.2 \%$. Intercuspidation on balancing side was found in $36.0 \pm 3.9 \%$ of persons who have physiological tooth wear, and $63.3 \pm 4.8 \%$ - pathological one $(\mathrm{p}<0.05)$. So, bilateral balancing occlusion promoted increased wear of occlusal surfaces, especially chewing segments of dentition.

As a conclusion, the mandatory annual prophylactic examination should provide for the detection of tooth wear, in addition to caries and periodontal diseases. It requires providing appropriate methodological support, in particular the establishment of clear differential clinical criteria for initial forms of tooth wear, and therefore indications for treatment. In addition, the need to develop and implement measures for primary prevention of the disease should be pointed out. 


\section{Fastovets Olena}

\section{References:}

1. Wetselaar P., Vermaire J. H., Visscher C.M., Lobbezoo F., \& Schuller A.A. (2016). The Prevalence of Tooth Wear in the Dutch Adult Population. Caries research, 50 (6): 543-550.

2. Luciano L.C.O., Ferreira M.C., \& Paschoal M.A. (2017). Prevalence and factors associated with dental erosion in individuals aged 12-30 years in a northeastern Brazilian city. Clinical, cosmetic and investigational dentistry, 9: 85-91.

3. Li M. H., \& Bernabé E. (2016). Tooth wear and quality of life among adults in the United Kingdom. Journal of Dentistry, 55: 48-53.

4. Muts E. J., van Pelt H., Edelhoff D., Krejci I., \& Cune M. (2014). Tooth wear: a systematic review of treatment options. The Journal of Prosthetic Dentistry, 112 (4): 752-759.

5. Abduo J., \& Lyons K. (2012). Clinical considerations for increasing occlusal vertical dimension: a review. Australian dental Journal, 57 (1): 2-10.

6. Bida V. I. (2002). Patolohichne styrannya tverdykh tkanyn zubiv ta osnovni pryntsypy yoho likuvannya [Pathological tooth wear and general principles of its treatment]. Kyiv: 96.

7. Shellis R. P., \& Addy M. P. (2014). The interactions between attrition, abrasion and erosion in tooth wear. Monographs in Oral Science, 25: 32-45.

8. Kontaxopoulou I., \& Alam S. (2015). Risk assessment for tooth wear. Primary dental journal, 4 (3): 25-29.

9. Wetselaar P., \& Lobbezoo F. (2016). The tooth wear evaluation system: a modular clinical guideline for the diagnosis and management planning of worn dentitions. Journal of Oral Rehabilitation, 43 (1): 69-80.

10. O'Toole S., Khan M., Patel A., Patel N. J., Shah N., Bartlett D., \& Movahedi S. (2018). Tooth wear risk assessment and care-planning in general dental practice. British Dental Journal, 224 (5): 73-85.

11. Mehta S.B., Banerji S., Millar B.J., \& Suarez-Feito J. M. (2012). Current concepts on the management of tooth wear: part 1. Assessment, treatment planning and strategies for the prevention and the passive management of tooth wear. British Dental Journal, 212 (1): 17-27.

12. Bartlett D. W., Lussi A., West N. X., Bouchard P., Sanz M., \& Bourgeois D. (2013). Prevalence of tooth wear on buccal and lingual surfaces and possible risk factors in young European adults. Journal of Dentistry, 41: 1007-1013.

13. Kazeko L. A., \& Kruglik O. A. (2009). Povyshennoye stiraniye zubov [Increased tooth wear]. Minsk: BSMU: 48.

14. Cunha-Cruz J., Pashova H., Packard J.D., Zhou L., \& Hilton T.J. (2010). Tooth wear: prevalence and associated factors in general practice patients. Community Dental Oral Epidemiology, 38 (3): 228-234.

15. Hvatova V. A. (2005). Klinicheskaya gnatologiya [Clinical gnathology]. Moscow, Meditsina, 2005: 296.

16. Klyomin V. A., Borisenko A. V., \& Ischenko P. V. (2004). Morfofunktsionalnaya i klinicheskaya otsenka zubov s defektami tverdyih tkaney [Morphofunctional and clinical estimation of teeth with defects of hard tissues]. Moscow: MEDpress-inform: 112. 